doi:10.17659/01.2017.0078

Journal of Case Reports 2017;7(3):293-295

\title{
Poor Supervision Leading to Carbon Monoxide Intoxication in a Burned Child
}

\author{
Chan Nie ${ }^{1}$, Tao Wang ${ }^{1}$, Xueqin Zeng ${ }^{2}$, Xiuquan Shi ${ }^{1,3}$ \\ ${ }^{1}$ Department of Epidemiology and Health Statistics, School of Public Health, Zunyi Medical University, Zunyi, Guizhou 563006, \\ China; ${ }^{2}$ Department of Burns and Plastic Surgery, Affiliated Hospital of Zunyi Medical University, Zunyi, Guizhou 563099, China; \\ ${ }^{3}$ Center for Injury Research and Policy \& Center for Pediatric Trauma Research, The Research Institute at Nationwide Children's \\ Hospital, The Ohio State University College of Medicine, Columbus, OH 43205, USA.
}

\begin{abstract}
Corresponding Author:
Dr. Xiuquan Shi

Email: xqshi2008@126.com licenses/by/3.0).

Received : August 8, 2017

Accepted : August 15, 2017

Published : August 20, 2017
\end{abstract}

This is an Open Access article distributed under the terms of the Creative Commons Attribution License (creativecommons.org/

\begin{abstract}
Background: We report this case to arouse public attention on burned children with carbon monoxide, (CO) intoxication, especially children under poor supervision and from coal high-consumption areas. Case Report: A eight-year old girl presented with $\mathrm{CO}$ intoxication and burned knee by a coal-burning stove. After hyperbaric oxygen to rectify CO intoxication and three surgeries therapy, the girl survived. Conclusion: Burns with $\mathrm{CO}$ intoxication are particularly dangerous for un-supervised children. Government and guardians should take some effective preventive measures and closer supervision to prevent childhood burns and other injuries.
\end{abstract}

Keywords: Accidents, Burns, Carbon Monoxide, Child, Coal, Hyperbaric Oxygenation.

\section{Introduction}

As one of the most devastating types of injuries, burns can be caused by various factors such as fire, boiled water, electricity and so on $[1,2]$. However, burns, which caused by a featured coal stove accompany with carbon monoxide (CO) intoxication is uncommon, especially some areas without district heat supply network. Guizhou province locates in southwest China; people still rely on coal for heating in winter particularly in the countryside.

There is a featured stove called "Huifenglu" that burned coal for room heating in Guizhou province. (Note: In Chinese, "Huifenglu" means a stove using coal which can warm up air, while cold air with oxygen can enter the stove from its bottom easily. It looks just like a table with a ferric surface). People can cook foods on the stove and sit around it to warm up in winter. Though this type of stoves have designed a chimney to exhaust the incomplete combustion gases outside of the room, the chimney is easily corroded by coal tar and then broken, while in most families, they change their chimney of Huifenglu at least for one year. Thus, $\mathrm{CO}$ poisoning incidents still occur occasionally. This paper reports a case of a little girl whose knee was roasted by the stove after she fainted by $\mathrm{CO}$ intoxication and lost her conscious for a long time, this aims to arouse public attention on burned children with $\mathrm{CO}$ intoxication, especially children under poor supervision and from coal high-consumption areas.

\section{Case Report}

An eight-year old girl, sat near the stove alone and did her homework at home with all windows and door closed. Then she felt difficulty in breathing and fainted, with her left knee roasted by the hot stove. After an undefined period of time (approximately 30 minutes), fortunately, she was found by her neighbors who rescued her out of the house and sent her to hospital. 
Ten hours later, the patient was admitted to the burn unit. At that time, she was almost revived by the fresh air except her lips was still cherry red. She presented a second degree deep burn located at the left knee [2\% of total body surface area, (TBSA)]. The wound had several large and small blisters formed, and had a black scab about $2 \times 4 \mathrm{~cm}$ diameters in the center. Around the trauma, part of the skin had taken off.

Based on the case history and clinical manifestations, she was diagnosed with left knee burn and $\mathrm{CO}$ intoxication. The patient was ECG (electrocardiogram) monitored and blood tests were performed. The heart rate was 125 beats/ min and blood pressure 114/89 mm Hg. No signs of organ (liver, kidney) dysfunction and inhalation injury were seen. Due to the CO poisoning history, hyperbaric oxygen therapy was conducted. Daily care dressing with burn ointment was offered on the wound. Then the patient was submitted to three surgical procedures: A surgical debridement and two-staged procedure (repairing of medial vascular network flap and skin grafting in left knee), with timely wound dressing was done. She was discharged home after one month without exudation or any discomfort.

A few days ago (after she left hospital for more than half a year), we made a follow-up to her. She had achieved complete recovery but was still little crippled in the burned leg.

\section{Discussion}

$\mathrm{CO}$ as a colorless, tasteless and odorless gas, produced by carbon or carbonaceous material combust insufficient in oxygen $[3,4]$, is often overlooked by people. It is toxic to multiple organs such as lung, brain, kidney and so on [5]. Even worse, severe $\mathrm{CO}$ poisoning can lead to death [6]. In Guizhou China, since it is not enough developed and there is a large number of coal mines, people rely on coal for heating in winter. So there is an urgent need to remind and alert local people to be cautious about burning coal. People should keep the air flowing, regularly check for the chimney leaks and windows and doors should not be closed. Moreover, those incomplete combustion gases can pollute air. So it is necessary for the government to make efforts and seek a more accessible alternative energy source to improve the local heating mode.

Burn area in this case seems not that severe, was only about $2 \%$ of TBSA, but it was located in the knee with a depth of second degree. Though three surgeries were conducted, she was finally crippled. It reminds us not to ignore a small area of burn, especially when it comes to joints at a great risk of deformities [7,8]. Immediate hospital intervention and receiving regular treatment helped her to prevent further possible deformities. Besides that, the hospital stay was almost one month, and the hospital cost was RMB 27,245 Yuan. Both of these important outcomes showed the severity of burns combined with $\mathrm{CO}$ intoxication. The father of the patient said that the medical insurance system in rural China should be appraised, which made a poor family in the countryside afford the expensive medical expenses.

In addition, guardians should be blamed for this accident for the most part. The little girl stayed at home without supervision, then caught $\mathrm{CO}$ intoxication and a second degree deep burn (it is more like a roast, just as a roast of sweet potato over gentle heat, which needs a long time). Finally, she was found by her neighbors, and her father and grandfather were busy on farm work in the field, while her parents divorced. Many parents consider that the best way to supervise their children is to keep them at home; as they believe it is safe enough for their children. However, in fact that is not the case. An open stove, kitchen knife, and electrical appliances all contribute to risk factors for children. Therefore, supervisors should not take the risk of leaving their little children at home alone for a long time. Unfortunately, there are many left-behind children in China $[9,10]$, especially in rural areas. 
Most farmers go to cities to make a living, and leave their children at home with their grandparents, who are too old to give a close and right supervision. As a result, rates of injuries among these vulnerable children increases. Fortunately, this problem has aroused public attention, but some effective measures remain lacking.

\section{Conclusion}

Burns and $\mathrm{CO}$ intoxication are preventable diseases. Government policy and family supervision play crucial roles in avoiding those accidents. Therefore, some preventive measures and closer supervision need to be taken to build a harmonious environment for children and keep them away from similar injuries.

Acknowledgments: We thank the girl and her father who agreed to participate in the study.

Contributors: $\mathrm{CN}$ : manuscript writing, literature search; TW, XZ: manuscript editing, literature search; XS: Study designing and manuscript editing. $\mathrm{CN}$ will act as guarantor. All authors approved the final version of manuscript.

Funding: This project was funded by the National Natural Science Foundation of China (No. 81560534, PI: Xiuquan Shi); Competing interests: None stated.

\section{References}

1. Trop M, Herzog SA, Pfurtscheller K, Hoebenreich AM, Schintler MV, Stockenhuber A, et al. The past 25 years of pediatric burn treatment in Graz and important lessons been learned. An overview. Burns. 2015;41:714-720.

2. Lee CJ, Mahendraraj K, Houng A, Marano M, Petrone
$\mathrm{S}$, Lee $\mathrm{R}$, et al. Pediatric burns: A single institution retrospective review of incidence, etiology, and outcomes in 2273 burn patients (1995-2013). J Burn Care Res. 2016;37:e579-e585.

3. Colakoglu S, Saritas A, Eroz R, Kandis H, Yaykasli KO, Akoz A, et al. Is one-time carbon monoxide intoxication harmless? Evaluation by argyrophilic nucleolarorganizing regions staining method. Hum Exp Toxicol. 2015;34:24-31.

4. Deniz T, Kandis H, Eroglu O, Gunes H, Saygun M. Carbon monoxide poisoning cases presenting with nonspecific symptoms. Toxicol Ind Health. 2017;33:53-60.

5. Kara H, Bayir A, Ak A, Degirmenci S. Cerebrovascular ischaemia after carbon monoxide intoxication. Singapore Med J. 2015;56:e26-28.

6. Dietz E, Gehl A, Friedrich P, Kappus S, Petter F, Maurer $\mathrm{K}$, et al. Carbon monoxide poisoning by a heating system. Arch Kriminol. 2016;237:93-101.

7. Ennis JL, Chung KK, Renz EM, Barillo DJ, Albrecht $\mathrm{MC}$, Jones JA, et al. Joint Theater Trauma System implementation of burn resuscitation guidelines improves outcomes in severely burned military casualties. J Trauma. 2008;64:S146-151.

8. Godleski M, Oeffling A, Bruflat AK, et al. Treating burn-associated joint contracture: results of an inpatient rehabilitation stretching protocol. J Burn Care Res. 2013;34:420-426.

9. Shen M, Yang S, Han J, Shi J, Yang R, et al. Non-fatal injury rates among the "left-behind children" of rural China. Inj Prev. 2009;15:244-247.

10. Shi S, Yang H, Hui Y, Zhou X, Wang T, Luo Y, et al. Epidemiologic characteristics, knowledge and risk factors of unintentional burns in rural children in Zunyi, Southwest China. Sci Rep. 2016;6:35445. 